\title{
CCL3LI gene copy number in individuals with and without HIV-associated neurocognitive disorder
}

This article was published in the following Dove Press journal:

Current Biomarker Findings

6 January 2012

Number of times this article has been viewed

\author{
Amanda Brown' \\ Ned Sacktor' \\ Karen Marder ${ }^{2}$ \\ Bruce Cohen ${ }^{3}$ \\ Giovanni Schifitto ${ }^{4}$ \\ Richard L Skolasky' \\ Jason Creighton' \\ Liping Guo' \\ Justin C McArthur'
}

'Department of Neurology, Johns Hopkins University School of Medicine, Baltimore, MD, ${ }^{2}$ Department of Neurology, Psychiatry, Sergievsky Center and Taub Institute on Alzheimers Disease and the Aging Brain, New York Presbyterian Hospital, Columbia University College of Physicians and Surgeons, New York, NY, ${ }^{3}$ Department of Neurology, Northwestern University Feinberg School of Medicine, Chicago, IL, ${ }^{4}$ Department of Neurology, University of Rochester, School of Medicine and Dentistry, Rochester, NY, USA
Correspondence: Amanda Brown Johns Hopkins University School of Medicine, Baltimore, 600 North Wolfe Street/Meyer 6-|8I, Baltimore, MD $2|287-7| 3 \mid$, USA

$\mathrm{Tel}+\mid 4106142429$

Fax +I 4I0 5026737

Email abrown76@jhmi.edu
Background: CCL3L1 copy number variation has been implicated as a marker for susceptibility and immunity to human immunodeficiency virus (HIV)-1 infection and its pathogenic sequelae. Some of these findings have been confirmed in several, but not all, subsequent independent cohort studies. A three-fold risk for the development of HIV-associated dementia was reported in individuals possessing a CCL3L1 copy number below the ethnic group median combined with a detrimental CCR5 genotype. With the availability of antiretroviral therapy since 1996, there has been a significant decline in HIV-associated dementia, and milder forms of HIV-associated neurocognitive impairment (HAND) are now most prevalent. Moreover, patients are living longer with HIV-1 infection and it is recognized that aging may be a contributory factor to the development of cognitive disorder. Thus, the need for biomarkers that can be used in clinical practice to identify and provide optimal treatment for those at increased risk for HAND is great. HAND affects 20\%-30\% of HIV-infected individuals, and several genetic loci which have been shown to confer susceptibility to HIV infection may also modulate the development of neurocognitive disorder. The aim of this study was to determine whether CCL3L1 chemokine gene copy number in selfdefined ethnic groups could differentiate HIV-infected individuals with and without HAND.

Methods: Genomic DNA was isolated from buccal swabs or peripheral blood mononuclear cells obtained from HIV-infected patients with or without a diagnoses of neurocognitive dysfunction in the Northeast AIDS Dementia Cohort and National NeuroAIDS Tissue Consortium. To maintain a uniform standard, a quantitative polymerase chain reaction design similar to previous studies using Taqman probes and fixed input DNA between $2 \mathrm{ng}$ and $10 \mathrm{ng}$ was used to determine a CCL3L1 copy number. Standard curves with two-fold dilutions from $25 \mathrm{ng}$ to $1.56 \mathrm{ng}$ were generated. CCL3L1 copy number was determined in triplicate in 262 subjects using quantitative polymerase chain reaction and the relative quantitation method. Data were analyzed using analysis of variance, with significance defined as $P<0.05$ and Bonferroni post hoc tests.

Results: Significant differences as determined by analysis of variance in CCL3L1 copy number between African-Americans and Caucasians $(P<0.0001)$ were found, highlighting ethnic group differences in the copy number of this gene. However, there were no differences in CCL3L1 copy number across the neurocognitive groups within each ethnic group. The median CCL3L1 copy number in African-Americans of two and Caucasians of one in this study was significantly lower than the previously reported ethnic group means of two and four copies, respectively. A higher prevalence of abnormal cognition with a relative risk of four was seen in African-Americans versus Caucasians.

Conclusion: Based on this nested case-control study, CCL3L1 copy number alone may not be useful for distinguishing between individuals at risk for mild or severe neurocognitive disorder. Additional larger cohort studies are required to determine whether CCL3L1 copy number in combination with polymorphisms in other genes known to contribute to HIV risk will be useful in identifying those at increased risk for HAND.

Keywords: neurological, HIV-associated dementia, HAND, chemokine, copy number, AfricanAmerican, Caucasian submit your manuscript | www.dovepress.com

Dovepress

http://dx.doi.org//0.2147/CBF.S27685
Current Biomarker Findings 2012:2 I-6

(C) 2012 Brown et al, publisher and licensee Dove Medical Press Ltd. This is an Open Access article which permits unrestricted noncommercial use, provided the original work is properly cited. 


\section{Introduction}

Human immunodeficiency virus (HIV)-associated neurocognitive disorders (HAND) remain among the most common clinical disorders encountered in people infected with HIV, even in an era in which combined antiretroviral therapy is widely deployed. HAND encompasses a hierarchy of progressively more severe patterns of central nervous system involvement, ranging from asymptomatic neurocognitive impairment, to mild neurocognitive disorder, to the more severe HIV-associated dementia. ${ }^{1}$ Asymptomatic neurocognitive impairment is the mildest form of HAND and is characterized by asymptomatic or unrecognized neurocognitive impairment. Individuals with asymptomatic neurocognitive impairment are more likely to develop more severe forms of HAND even under conditions of effective virus suppression. ${ }^{1} \mathrm{HIV}$-associated dementia represents the most severe form of HAND, with significant functional impairments and is synonymous with HIV encephalopathy and autoimmune deficiency syndrome (AIDS) dementia complex. Since the introduction of combined antiretroviral therapy in 1996, many combined antiretroviral therapytreated patients have shown durable and complete suppression of HIV replication. The incidence of moderate to severe dementia fell from about $7 \%$ in 1989 to only $1 \%$ in 2000 , and the severity of neurological disease appears to have been attenuated. ${ }^{2,3}$ Despite this remarkable effect on incidence rates, the prevalence of HAND continues at very high rates between $15 \%$ and $50 \%{ }^{4}$ For example, in the CHARTER (CNS HIV Antiretroviral Therapy Effects Research) cohort, $53 \%$ of the total sample had neurocognitive impairment, with increasing rates in those groups with greater comorbid illnesses. ${ }^{5}$

The continued high prevalence of HAND in combined antiretroviral therapy-treated individuals suggests that HIVrelated sequelae remain a problem. Moreover, a significant number of individuals with mild neurocognitive impairment often progress to more severe disease. In this regard, recent studies have shown that there is wide variability in the penetration of antiretrovirals into the brain and, in those individuals receiving drugs that effectively enter the central nervous system, the risk of HAND is significantly decreased. ${ }^{6,7}$ Given the fact that only a subset of all HIVinfected individuals develop HAND, genetics is likely to play a key role in determining susceptibility to neurologic disease, and hence the ability to identify those HIV-infected individuals at increased risk for HAND would allow clinicians to design the most effective combined antiretroviral therapy regimens to prevent progression of neurocognitive disorder. In this regard, a panel of validated predictive markers would serve as invaluable tools for identifying those individuals at risk for the development of HAND and the study of such factors would also increase our understanding of their role in HIV neuropathogenesis.

CCL3L1 is the most potent ligand for the HIV-coreceptor CCR5 and chemoattractant for macrophages. ${ }^{8}$ Activated HIV-infected and uninfected macrophages play a central role in the development of HIV-associated neurocognitive dysfunction through the secretion of inflammatory cytokines and neurotoxic factors. ${ }^{9,10}$ Indeed, in vitro studies have shown that cells with higher CCL3L1 ligand levels can bind to CCR5 and protect cells from HIV infection. ${ }^{8,11-13}$ Several gene loci involved in immune responses and human disease are subject to segmental gene duplication. Such duplications, which occur in $5 \%-15 \%$ of the human genome can be inherited, occur de novo, and make significant contributions to human genomic variation. ${ }^{14}$ The CCL3L1 locus on chromosome 17q11.2-q12 is a hotspot for segmental gene duplications ${ }^{15-17}$ and significant copy number differences exist amongst ethnic groups. ${ }^{18,19} \mathrm{In}$ this regard, Gonzalez et al reported that a median CCL3L1 chemokine gene copy number below the ethnic group average in combination with CCR5 detrimental alleles increased the risk for HIV infection and the development of key AIDS-defining illnesses, including HIV-associated dementia. ${ }^{18}$ Interestingly, a recent study using in vitro cultured neurons demonstrated that CCL3L1 can protect neurons from HIV Env-induced cell death, ${ }^{20}$ suggesting the possibility that possession of a high CCL3L1 copy number and increased production of this chemokine ligand could hinder the development of HIV-associated cognitive impairment. Using DNA samples obtained from a cohort of HIV-infected persons with advanced disease, we tested the hypothesis that CCL3L1 copy number is associated with the development of HAND.

\section{Methods and data analysis}

This nested case-control study included buccal swab samples from individuals from the Northeast AIDS Dementia Cohort (NEAD), which was composed of patients with advanced disease from four participating sites using protocols approved by the institutional review boards of Johns Hopkins University School of Medicine, University of Rochester, Columbia University, and Northwestern University. ${ }^{21,22}$ Patients consenting to have buccal swabs 
taken at the baseline visit were informed in the consent form that the DNA would be used to study genetic risk factors associated with HIV neurological disease, would be stored nonidentified, used only for research purposes, and not be part of routine clinical care. Specifically individuals were recruited if they had a CD4 count $<200$ or had neurocognitive symptoms and CD4 count $<300$. Genomic DNA from HIV-infected patients with stable neurologic diagnosis at two visits were included. In addition, DNA samples from the National NeuroAIDS Tissue Consortium (NNTC) were also analyzed. ${ }^{23}$ Psychiatric diagnoses of individuals in the NNTC cohort were determined using the Psychiatric Research Interview for Substance and Mental Disorders (PRISM) or by Composite International Diagnostic Interview (CIDI). Both PRISM and CIDI assign psychiatric diagnoses according to the Diagnostic and Statistical Manual of Mental Disorders-IV. For the NEAD cohort, subjects were categorized as normal, minor cognitive motor disorder (MCMD), or HIV dementia using American Association for Neurology criteria prior to establishment of the asymptomatic neurocognitive impairment category. ${ }^{1}$ The Memorial Sloan Kettering (MSK) classification was used to determine the severity of dementia. ${ }^{24}$ The equivalent neurocognitive diagnoses between MSK and the NNTC (PRISM or CIDI) are MSK $0=$ neurocognitively normal, MSK $0.5=$ subsyndromic, MSK 1.0 = possible or probable MCMD, and MSK 2 to $4=$ possible or probable HIV-associated dementia dependingupon severity and wasutilized to group the corresponding patient DNA samples. For this study, MSK 0.5 was categorized as MCMD, and MSK 1 or above was designated as HIV-associated dementia.

Genomic DNA was isolated from buccal swabs or PBMCs using the QIAamp DNA Mini Kit (Qiagen, Valencia, CA) as described by the manufacturer. Typical yields from a single swab that had been stored for a year or more at $-70^{\circ} \mathrm{C}$ ranged from 20 to $50 \mathrm{ng} / \mu \mathrm{L}$ in a total volume of $140-145 \mu \mathrm{L}$. DNAs were diluted before amplification to 5-10 ng/reaction. The experimental and quantitative methods for determining the CCL3L1 copy number were based on the study by Gonzalez et al. ${ }^{18}$ The primer sequences for CCL3L1 were 5'TCTCCACAGCTTCCTAACCAAGA, 5'CTGGACCCACTCCTCACTGG (Invitrogen, Carlsbad, CA) and probe, VIC-AGGCCGGCAGGTCTGTGCTGATAMARA. The housekeeping gene, $\beta$-globin was amplified with the primers and probe, 5'GGCAACCCTAAGGTGAAGGC, 5'GGTGAGCCAGGCCATCACTA and 6-FAMCATGGCAAGAAAGTGCTCGGTGCCT-TAMARA.
The final concentrations of primer and probe in the reactions were 900 and $250 \mathrm{nM}$, respectively, in the presence of Taq master mix (Eurogentec or Applied Biosystems). The polymerase chain reaction (PCR) conditions were one cycle, $50^{\circ} \mathrm{C}$ and 2 minutes; one cycle, $95^{\circ} \mathrm{C}$ and 10 minutes; 40 cycles at $95^{\circ} \mathrm{C}$ and 15 seconds, $60^{\circ} \mathrm{C}$ and one minute. The sample DNAs (5-10 ng/reaction) were run in triplicate on the ABI Prism 7000 Sequence Detection System (Applied Biosystems, Foster City, CA). Serial dilutions of genomic DNA from A431 cells (25-1.56 ng, BioChain Institute Inc, Hayward, CA), which have been shown to have two copies of CCL3L1 per diploid genome were used to generate standard curves of the threshold cycle $\left(\mathrm{C}_{\mathrm{t}}\right)$ against the $\log$ [DNA] for each 96-well plate. Any samples that did not fall within the standard curve with $\mathrm{R}^{2}$ of at least 0.96 were repeated. Data were analyzed using one-way analysis of variance, and with Bonferroni post hoc tests (GraphPad Prism).

\section{Results}

In this nested case-control study, CCL3L1 copy number was determined in a total of 262 samples, comprising 158 AfricanAmericans and 104 Caucasians who were distributed among four neurocognitive groups (Table 1). A one-way analysis of variance between African-Americans and Caucasian subjects in the different neurocognitive groups revealed a significant difference in CCL3L1 median copy number between groups $[\mathrm{F}(7,254)=6.11 ; P \leq 0.0001]$. Post hoc analyses with $\alpha=0.05$ ( $95 \%$ confidence) using the Bonferroni test was used to examine intragroup variation. A significant difference in CCL3L1 mean copy number between African-Americans (mean $=2.42$, standard deviation $[\mathrm{SD}]=1.56)$ and Caucasians $($ mean $=1.32, \mathrm{SD}=0.961)$ with $\mathrm{MSK}=0(P<0.05 ; 95 \%$ CI 0.204-2) or $\mathrm{MSK}=2$ (African-Americans: mean $=2.55$, $\mathrm{SD}=0.816$; Caucasians: mean $=1.03, \mathrm{SD}=0.352 ; P<0.05$; 95\% CI 1.51-3.34) was found. No significant differences within ethnic groups in the CCL3L1 mean copy number was found across cognitive diagnoses. In the absence of HIVnegative controls with similar demographic backgrounds as found in the NEAD and NNTC cohorts we used one-way analysis of variance to compare the median CCL3L1 copy number between African-Americans and Caucasians against the previously reported median copy number for these groups that was obtained from 675 Caucasians and 498 AfricanAmericans, where the median values of four for AfricanAmericans and two for Caucasians were ascertained. ${ }^{18}$ In all cognitive groups within African-Americans or 
Table I Lack of association between CCL3LI copy number and HIV-associated neurocognitive diagnosis

\begin{tabular}{|c|c|c|c|c|c|c|c|c|c|c|c|}
\hline \multirow[t]{2}{*}{ Ethnic group } & \multirow[t]{2}{*}{$\begin{array}{l}\text { Mean age } \\
\text { (SD) }\end{array}$} & \multirow[t]{2}{*}{$\begin{array}{l}\text { Neurocognitive } \\
\text { diagnosis }\end{array}$} & \multirow[t]{2}{*}{$\mathbf{N}$} & \multirow[t]{2}{*}{ Mean } & \multirow[t]{2}{*}{ SD } & \multirow[t]{2}{*}{$\begin{array}{l}95 \% \text { confidence } \\
\text { interval }\end{array}$} & \multicolumn{2}{|c|}{$\begin{array}{l}\text { Interquartile } \\
\text { range }\end{array}$} & \multirow[t]{2}{*}{$\begin{array}{l}\text { Median } \\
\text { (rounded) }\end{array}$} & \multirow[t]{2}{*}{ Min } & \multirow[t]{2}{*}{ Max } \\
\hline & & & & & & & $25 \%$ & $75 \%$ & & & \\
\hline \multirow[t]{4}{*}{ African-American } & $46.4(7.11)$ & MSK 0 & 29 & 2.42 & 1.56 & $1.82-3$ & 1.22 & 2.95 & 2 & 0.697 & 6.67 \\
\hline & $46.3(7.75)$ & MSK 0.5 & 59 & 2.49 & 1.34 & $2.14-2.84$ & 1.51 & 3.1 & 2 & 0.575 & 7.23 \\
\hline & $46.2(6.17)$ & MSK I & 43 & 2.33 & 1.25 & $|.94-2.7|$ & 1.39 & 2.68 & 2 & 0.679 & 5.69 \\
\hline & $46.2(7.95)$ & MSK 2 & 27 & 2.55 & 0.816 & $2.22-2.87$ & 1.86 & 3.14 & 2 & 1.12 & 4.27 \\
\hline \multirow[t]{4}{*}{ Caucasian } & $49.5(7.63)$ & MSK 0 & 51 & 1.32 & 0.961 & $1.05-1.59$ & 0.742 & 1.47 & 1 & 0.531 & 5.47 \\
\hline & 42.4 (8.99) & MSK 0.5 & 12 & 1.79 & 1.22 & $1.02-2.56$ & 0.709 & 2.6 & 1 & 0.579 & 4.22 \\
\hline & $44.4(6.83)$ & MSK I & 31 & 1.98 & 1.41 & $1.46-2.49$ & 1 & 2.58 & I & 0.606 & 6.53 \\
\hline & $4 I .8(8.28)$ & MSK 2 & 10 & 1.03 & 0.352 & $0.783-1.29$ & 0.739 & 1.31 & I & 0.583 & I.7I \\
\hline
\end{tabular}

Abbreviations: SD, standard deviation; MSK, Memorial Sloan Kettering score.

Caucasians, there was a significant $(P<0.0001)$ difference in the median CCL3L1 copy numbers in the combined NEAD/ NNTC cohort compared with the previously reported medians for these ethnic groups. ${ }^{18}$ Notably, the prevalence of normal cognition in the combined NEAD/NNTC cohort was $18 \%$ for African-Americans and 49\% for Caucasians (Table 1). The relative risk of HIV-associated neurocognitive disorder for African-Americans in this cohort was 4.28 compared with Caucasians. There were no significant differences in mean age between ethnic groups with the same neurocognitive diagnosis (Table 1).

\section{Discussion}

We were intrigued with the previously reported finding of a three-fold increased risk of developing HIV-associated

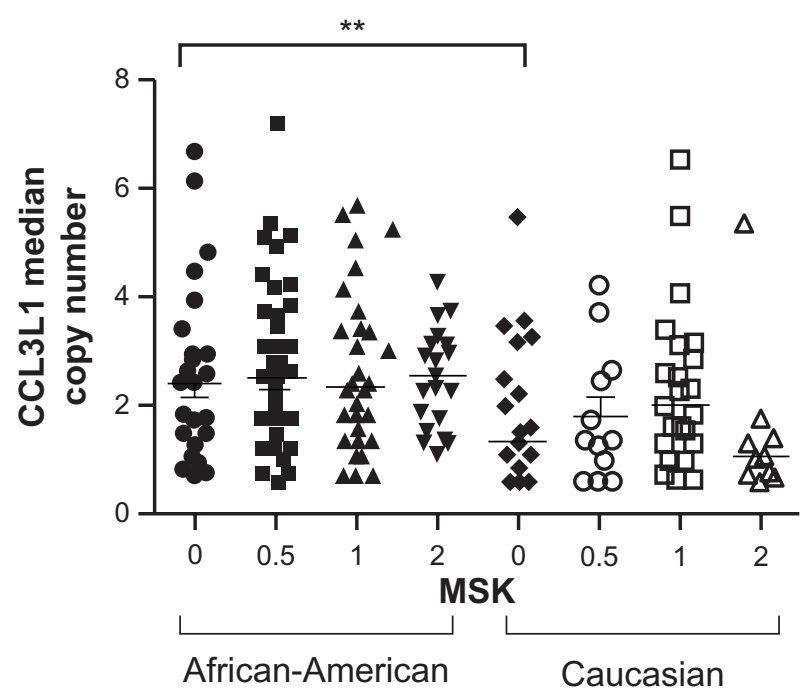

Figure I Significant difference in CCL3LI copy number in African-Americans compared with Caucasians.

Notes: The median CCL3LI copy number among HIV-infected African-Americans and Caucasians stratified by severity of cognitive impairment using the Memorial Sloan Kettering score (MSK); MSK 0, cognitively normal; MSK 0.5, mild impairment; MSK I-2, severe dementia. dementia in individuals with CCL3L1 median copy numbers below the ethnic group average combined with CCR5 detrimental genotypes. ${ }^{18}$ While our study did confirm previous reports of median CCL3L1 copy number differences between African-American and Caucasian ethnic groups, we did not find any association between CCL3L1 median copy number in HIV-infected individuals with or without neurocognitive impairment. Several explanations may account for our inability to detect an association. Firstly, however, our study was not grossly underpowered, because statistical analyses suggested that an additional 350 subjects in each group to detect an effect size of 0.25 units of deviation would have identified a tiny statistical, but not clinically significant difference. Secondly, the composition of the cohorts may have had an impact on our ability to detect an association. In contrast with the racially balanced prospective cohort used in the Gonzalez et al study, ${ }^{18}$ made up of US air force personnel who developed HIV infection, the NEAD cohort established in 1998 is composed predominantly of AfricanAmerican individuals with advanced HIV disease who have in many cases had poor access to health care, issues with adherence to treatment regimens, and illicit drug use..$^{25}$ The NNTC cohort established in 1998 collects and stores tissue samples, as well as clinical and neuropsychological testing data on HIV-infected individuals with advanced disease..$^{23,26}$ In this regard, we found that unimpaired Caucasian individuals had a median copy number of one and African-Americans of two, which were significantly lower than the two and four copy average, respectively, previously reported in healthy individuals from these groups. ${ }^{18}$ Thirdly, the Gonzalez et al study used outcome measures of the pre-combined antiretroviral therapy era, such as AIDS-defining illnesses. In contrast, the majority of the patients used in this study were on long-term antiretroviral therapy that was effective in reducing most AIDS-defining illnesses, including severe 
forms of HIV-associated dementia. A possible limitation of our study is that we did not account for differences in the duration of HIV infection and length of antiviral therapy. Our quantitative PCR assay possessed the dynamic range to detect a wide variation in CCL3L1 copy numbers and therefore negating such limitations that have been raised as issues in other studies. ${ }^{27}$ Furthermore, Gonzalez et al showed an interaction between CCR5 genotypes and CCL3L1, which is one of several ligands including MCP-1 and RANTES that bind to this receptor. In this study, we did not examine the CCR5 genotype. However, we have previously shown that increased MCP-1 in the cerebrospinal fluid is associated with HIV-associated dementia. ${ }^{28}$ Interestingly, a recent study in a Chinese cohort did not find any association between CCL3L1 copy number and neurocognitive impairment. ${ }^{29}$

In this study, we found a significant difference in the prevalence of normal cognition and a four-fold increased risk for neurocognitive dysfunction in HIV-infected AfricanAmericans versus Caucasians. Early studies examining progression of neurocognitive disorder in the pre-combined antiretroviral therapy era found that a history of injection drug use, but not race, gender, or age, was associated with more rapid neurologic progression. ${ }^{30}$ In this regard, individuals in the NEAD cohort do have a higher incidence of drug abuse, ${ }^{21}$ and in combination with adherence and health care access issues, may be contributing factors leading to ethnic differences in neurocognitive dysfunction.

Antiretroviral therapy has led to a significant reduction in the occurrence of HIV-associated neurocognitive disorder however milder forms of the disease continue to increase in prevalence and incidence. In part, such changes may be associated with the aging of HIV-infected individuals who have been on long-term antiviral therapy. ${ }^{31,32}$ Indeed, recent imaging studies suggest that aging of the brains of HIV-infected individuals is greatly accelerated compared with uninfected persons of similar age. ${ }^{33}$ In order to improve therapeutic interventions, a panel of biomarkers to detect individuals at risk for the development of HAND is needed. The CCL3L1 copy number may be useful in detecting individuals more likely to display increased susceptibility to HIV and its effects on the immune system. ${ }^{34}{ }^{41}$ Although this finding has been questioned, ${ }^{42,43}$ its utility as a predictive marker for HAND in the era of combined antiretroviral therapy is as yet unclear. Additional large cohort studies to determine whether CCL3L1 copy number in combination with polymorphisms in other genes known to contribute to HIV risk can be used to distinguish between those at risk for mild or severe neurocognitive disorder are required. ${ }^{38,44}$

\section{Acknowledgments}

We acknowledge the generous participation of patients and staff of the NEAD cohort and the NNTC, and our funding sources (NIH R01NS049465, PO30MH075673, N01MH32002, and ULIRR024156).

\section{Disclosure}

The authors declare that they have no competing interests in this work.

\section{References}

1. Antinori A, Arendt G, Becker JT, et al. Updated research nosology for HIV-associated neurocognitive disorders. Neurology. 2007;69: 1789-1799.

2. Dore GJ, McDonald A, LiY, Kaldor JM, Brew BJ. Marked improvement in survival following AIDS dementia complex in the era of highly active antiretroviral therapy. AIDS. 2003;17:1539-1545.

3. Sacktor N, McDermott MP, Marder K, et al. HIVassociated cognitive impairment before and after the advent of combination therapy. J Neurovirol. 2002;8:136-142.

4. Clifford DB. HIV-associated neurocognitive disease continues in the antiretroviral era. Top HIV Med. 2007;16:94-98.

5. Heaton RK, Franklin DR, Ellis RJ, et al. HIV-associated neurocognitive disorder before and during the era of combination antiretroviral therapy: differences in rates, nature and predictors. J Neurovirol. 2011;17: 3-16.

6. Letendre S, Marquie-Beck J, Capparelli E, et al. Validation of the CNS penetration-effectiveness rank for quantifying antiretroviral penetration into the central nervous system. Arch Neurol. 2008;65:65-70.

7. Best BM, Letendre SL, Brigid E, et al. Low atazanavir concentrations in cerebrospinal fluid. AIDS. 2009;23:83-87.

8. Menten P, Struyf S, Schutyser E, et al. The LD78beta isoform of MIP1alpha is the most potent CCR5 agonist and HIV-1-inhibiting chemokine. J Clin Invest. 1999;104:R1-R5.

9. Roberts TK, Buckner CM, Berman JW. Leukocyte transmigration across the blood-brain barrier: perspectives on neuroAIDS. Front Biosci. 2010; 15:478-536.

10. Kraft-Terry SD, Stothert AR, Buch S, Gendelman HE. HIV-1 neuroimmunity in the era of antiretroviral therapy. Neurobiol Dis. 2010;37: 542-548.

11. Xin X, Shioda T, Kato A, Liu H, Sakai Y, Nagai Y. Enhanced antiHIV-1 activity of CC-chemokine LD78beta, a non-allelic variant of MIP-1alpha/LD78alpha. FEBS Lett. 1999;457:219-222.

12. Nibbs RJ, Yang J, Landau NR, Mao JH, Graham GJ. LD78beta, a non-allelic variant of human MIP-1alpha (LD78alpha), has enhanced receptor interactions and potent HIV suppressive activity. J Biol Chem. 1999;274:17478-17483.

13. Aquaro S, Menten P, Struyf S, et al. The LD78beta isoform of MIP-1alpha is the most potent CC-chemokine in inhibiting CCR5 dependent human immunodeficiency virus type 1 replication in human macrophages. J Virol. 1999;75:4402-4406.

14. Colobran R, Pedrosa E, Carretero-Iglesia L, Juan M. Copy number variation in chemokine superfamily: the complex scene of CCL3L-CCL4L genes in health and disease. Clin Exp Immunol. 2010;162:41-52.

15. Townson JR, Barcellos LF, Nibbs RJB. Gene copy number regulates the production of the human chemokine CCL3-L1. Eur J Immunol. 2002;32:3016-3026.

16. Nakao M, Nomiyama H, Shimada K. Structures of human genes coding for cytokine LD78 and their expression. Mol Cell Biol. 1990;10: 3646-3658.

17. Irving S, Zipfel P, Balke J, et al. Two inflammatory mediator cytokine genes are closely linked and variably amplified on chromosome 17q. Nucleic Acids Res. 1990;18:3261-3270. 
18. Gonzalez E, Kulkarni H, Bolivar $\mathrm{H}$, et al. The influence of CCL3L1 gene-containing segmental duplication on HIV-1/AIDS susceptibility. Science. 2005;307:1434-1440.

19. Colobran R, Comas D, Faner R, et al. Population structure in copy number variation and SNPs in the CCL4L chemokine gene. Genes Immun. 2008:279-288.

20. Chun H, Hao W, Honghai Z, Ning L, Yasong W, Chen D. CCL3L1 prevents gp120-induced neuron death via the CREB cell signaling pathway. Brain Res. 2009;1257:75-88.

21. Sacktor N. The epidemiology of human immunodeficiency virusassociated neurological disease in the era of highly active antiretroviral therapy. J Neurovirol. 2002;8:115-121.

22. Marder K, Albert SM, McDermott MP, et al. Inter-rater reliability of a clinical staging of HIV-associated cognitive impairment. Neurology. 2003;60:1467-1473.

23. Morgello S, Gelman BB, Kozlowski PB, et al. The National NeuroAIDS Tissue Consortium: a new paradigm in brain banking with an emphasis on infectious disease. Neuropathol Appl Neurobiol. 2001; 27:326-335.

24. Price RW, Brew BJ. The AIDS dementia complex. J Infect Dis. 1988; 158:1079-1083.

25. Sacktor N, McDermott MP, Marder K, et al. HIV-associated cognitive impairment before and after the advent of combination therapy. J Neurovirol. 2002;8:136-142.

26. Everall I, Vaida F, Khanlou N, et al. Cliniconeuropathologic correlates of human immunodeficiency virus in the era of antiretroviral therapy. J Neurovirol. 2009;15:360-370.

27. Shrestha S, Nyaku M, Edberg J. Variations in CCL3L gene cluster sequence and non-specific gene copy numbers. BMC Res Notes. 2010; 3:74.

28. Kelder W, McArthur JC, Nance-Sproson T, McClernon D, Griffin DE. Beta-chemokines MCP-1 and RANTES are selectively increased in cerebrospinal fluid of patients with human immunodeficiency virusassociated dementia. Ann Neurol. 1998;44:831-835.

29. Spector SA, Singh KK, Gupta S, et al. APOE epsilon4 and MBL-2 $\mathrm{O} / \mathrm{O}$ genotypes are associated with neurocognitive impairment in HIVinfected plasma donors. AIDS. 2010;24:1471-1479.

30. Bouwman FH, Skolasky RL, Hes D, et al. Variable progression of HIVassociated dementia. Neurology. 1998;50:1814-1820.

31. Valcour V, Watters MR, Williams AE, Sacktor N, McMurtray A, Shikuma C. Aging exacerbates extrapyramidal motor signs in the era of highly active antiretroviral therapy. J Neurovirol. 2008;14:362-367.
32. Nath A, Schiess N, Venkatesan A, Rumbaugh J, Sacktor N, McArthur JC. Evolution of HIV dementia with HIV infection. Int Rev Psychiatry. 2008;20:25-31.

33. Ances BM, Vaida F, Yeh MJ, et al. HIV infection and aging independently affect brain function as measured by functional magnetic resonance imaging. J Infect Dis. 2010;201:336-340.

34. Kulkarni H, Marconi VC, Agan BK, et al. Role of CCL3L1-CCR5 genotypes in the epidemic spread of HIV-1 and evaluation of vaccine efficacy. PLoS One. 2008;3:e3671.

35. Kulkarni H, Agan BK, Marconi VC, et al. CCL3L1-CCR5 genotype improves the assessment of AIDS Risk in HIV-1-infected individuals. PLoS One. 2008;3:e3165.

36. Dolan M, Kulkarni H, Camargo J, et al. CCL3L1 and CCR5 influence cell-mediated immunity and affect HIV-AIDS pathogenesis via viral entry-independent mechanisms. Nat Immunol. 2007;8:1324-1336.

37. Ahuja SK, Kulkarni H, Catano G, et al. CCL3L1-CCR5 genotype influences durability of immune recovery during antiretroviral therapy of HIV-1-infected individuals. Nat Med. 2008;14:413-420.

38. Shostakovich-Koretskaya L, Catano G, Chykarenko ZA, et al. Combinatorial content of CCL3L and CCL4L gene copy numbers influence HIV-AIDS susceptibility in Ukrainian children. AIDS. 2009; 23:679-688

39. Shalekoff S, Meddows-Taylor S, Schramm DB, et al. Host CCL3L1 gene copy number in relation to HIV-1-specific CD4+ and CD8+ T-cell responses and viral load in South African women. $J$ Acquir Immune Defic Syndr. 2008;48:245-254.

40. Meddows-Taylor S, Donninger SL, Paximadis M, et al. Reduced ability of newborns to produce CCL3 is associated with increased susceptibility to perinatal human immunodeficiency virus 1 transmission. J Gen Virol. 2006;87:2055-2065.

41. Kuhn L, Schramm DB, Donninger SL, et al. African infants' CCL3 gene copies influence perinatal HIV transmission in the absence of maternal nevirapine. AIDS. 2007;21:1753-1761.

42. Urban TJ, Weintrob AC, Fellay J, et al. CCL3L1 and HIV/AIDS susceptibility. Nat Med. 2009;15:1110-1112.

43. Bhattacharya T, Stanton J, Kim EY, et al. CCL3L1 and HIV/AIDS susceptibility. Nat Med. 2009;15:1112-1115.

44. Salgado M, Simón A, Sanz-Minguela B, et al. An additive effect of protective host genetic factors correlates with HIV nonprogression status. J Acquir Immune Defic Syndr. 2011;56:300-305.
Current Biomarker Findings

\section{Publish your work in this journal}

Current Biomarker Findings is an international, peer-reviewed, open access journal publishing original research, reports, reviews and commentaries on all areas of biomarker research. The manuscript management system is completely online and includes a very quick and fair

\section{Dovepress}

peer-review system. Visit http://www.dovepress.com/testimonials.php to read real quotes from published authors. 